Check for updates

Cite this: Chem. Commun., 2020, 56, 3681

Received 12th December 2019, Accepted 28th January 2020

DOI: $10.1039 / \mathrm{c} 9 \mathrm{cc} 09638 \mathrm{~h}$

rsc.li/chemcomm

\section{Validation of the inverted adsorption structure for free-base tetraphenyl porphyrin on $\mathrm{Cu}(111) \dagger$}

\author{
P. T. P. Ryan, (D) ${ }^{a b}$ P. L. Lalaguna, ${ }^{a c}$ F. Haag, ${ }^{d}$ M. M. Braim, ${ }^{e}$ P. Ding, (D) ef \\ D. J. Payne, (D) ${ }^{\text {b J. V. Barth, }}{ }^{d}$ T.-L. Lee, ${ }^{a}$ D. P. Woodruff, (D) ${ }^{e}$ F. Allegretti (D) ${ }^{d}$ and \\ D. A. Duncan (D)*a
}

\begin{abstract}
Utilising normal incidence $X$-ray standing waves we rigourously scrutinise the "inverted model" as the adsorption structure of freebase tetraphenyl porphyrin on $\mathrm{Cu}(111)$. We demonstrate that the iminic $\mathrm{N}$ atoms are anchored at near-bridge adsorption sites on the surface displaced laterally by $1.1 \pm 0.2 \AA$ in excellent agreement with previously published calculations.
\end{abstract}

Porphyrins are an important class of macrocyclic compounds with a wide variety of electronic and chemical properties that have led them to be widely studied in a variety of fields in materials science. In particular, on surfaces of metals and metal oxides, tetraphenyl porphyrins have been extensively studied (e.g. ref. 1-4 and citations therein) as prototypical porphyrin species. The free-base tetraphenyl porphyrin, $2 H$-TPP (shown schematically in Fig. 1a), exhibits two chemically distinct $\mathrm{N}$ species in X-ray photoelectron spectroscopy (XPS), ${ }^{5}$ which are assigned to the iminic $(-\mathrm{N}=)$ and aminic $\left(-\mathrm{NH}^{-}\right)$nitrogen atoms. For many years $2 H$-TPP was assumed to adsorb on almost all metal surfaces in a saddle-shaped conformation, ${ }^{5-7}$ in which two of the four pyrrole groups tilt towards, and the other two tilt away, from the surface plane, thus allowing the planes of the phenyl groups to rotate away from an orientation perpendicular to the surface, the expected conformation of the free molecule. Recent work by Lepper et al. ${ }^{8}$ Albrecht et al. ${ }^{9}$ and Moreno-López et al. ${ }^{10}$ however, have proposed a so-called inverted model to describe the conformation of the dominant species on $\mathrm{Cu}(111)$, whereby the two

\footnotetext{
${ }^{a}$ Diamond Light Source, Harwell Science and Innovation Campus, Didcot, OX11 OQX, UK. E-mail: david.duncan@diamond.ac.uk

${ }^{b}$ Department of Materials, Imperial College London, SW7 2AZ, UK

${ }^{c}$ School of Chemistry, University of Glasgow, G12 8QQ, UK

${ }^{d}$ Physics Department E20, Technical University of Munich, James Franck Straße 1, D-85748 Garching, Germany

${ }^{e}$ Department of Physics, University of Warwick, Coventry, CV4 7AL, UK

${ }^{f}$ State Key Laboratory of Urban Water Resource and Environment,

School of Chemistry and Chemical Engineering, Harbin Institute of Technology, Harbin 150001, China

$\dagger$ Electronic supplementary information (ESI) available: Further experimental details, supporting figures and tables. See DOI: 10.1039/c9cc09638h
}

iminic pyrrole groups are rotated to be perpendicular to the surface, breaking the aromaticity of the macrocycle. The pertaining results of density functional theory (DFT) calculations indicate that in the saddle conformation the local bonding of the iminic $\mathrm{N}$ atoms is off-atop $\mathrm{Cu}$ sites on the surface, but, in the inverted conformation, these $\mathrm{N}$ atoms are instead located in $\mathrm{Cu}$ bridging sites, as shown schematically in Fig. S1a and $\mathrm{c}$ in the ESI. $\dagger$ The main evidence supporting this model is based on the comparison of measured STM images and simulated images from DFT calculations, despite the well-known associated uncertainty arising from studies based on these two techniques alone (e.g. ref. 11 and 12). However, Lepper $e t a l .^{8}$ also showed that the adsorption heights of the two chemically distinct $\mathrm{N}$ species of $2 \mathrm{H}$-TPP adsorbed on $\mathrm{Cu}(111)$, predicted by their DFT calculations for the inverted model, agreed well with published quantitative structural measurements of these parameters by Bürker et al. ${ }^{13}$ using normal incidence X-ray standing waves (NIXSW). ${ }^{14}$ By contrast these measured heights are much less consistent with those of the calculated saddle-shape model. However, Bürker et al. do report severe radiation damage during their measurement, which had an unknown effect on the adsorbed species, but was assigned to photon induced $\mathrm{Cu}$-metallation; their results also provided a measure only of the height of the $\mathrm{N}$ atoms, but not of their lateral registry relative to the underlying $\mathrm{Cu}(111)$ surface. Additionally, an earlier $\mathrm{N}$ K-edge and $\mathrm{C}$ K-edge near edge X-ray absorption fine structure (NEXAFS) study ${ }^{5}$ into a monolayer of $2 H$-TPP on $\mathrm{Cu}(111)$ indicated a tilt of the iminic pyrrole of only $60^{\circ}$, with a comparable tilt of the aminic pyrrole of $40^{\circ}$ (inverted model: $80^{\circ}$ and $10^{\circ}$, respectively), though the interpretation of these NEXAFS spectra is complicated by the potential hybridisation of the $\mathrm{Cu}$ and porphyrin orbitals, as is observed for porphine $(2 H-\mathrm{P})^{15}$ (discussed further in the ESI $\dagger$ ). Moreover, our prior energy-scanned photoelectron diffraction (PhD) study into $2 H-\mathrm{P}$ on this surface found the lateral adsorption site of the iminic $\mathrm{N}$ atoms to be off-atop (see Fig. S1b and d, ESI $\dagger$ ) with the adsorption heights of the aminic and iminic $\mathrm{N}$ atoms similar to those of the $\mathrm{N}$ atoms of $2 \mathrm{H}$-TPP on $\mathrm{Cu}^{16}$ This work demonstrates that the porphine macrocycle can indeed adsorb at a site with short $\mathrm{N}-\mathrm{Cu}$ adsorption heights, 
a)
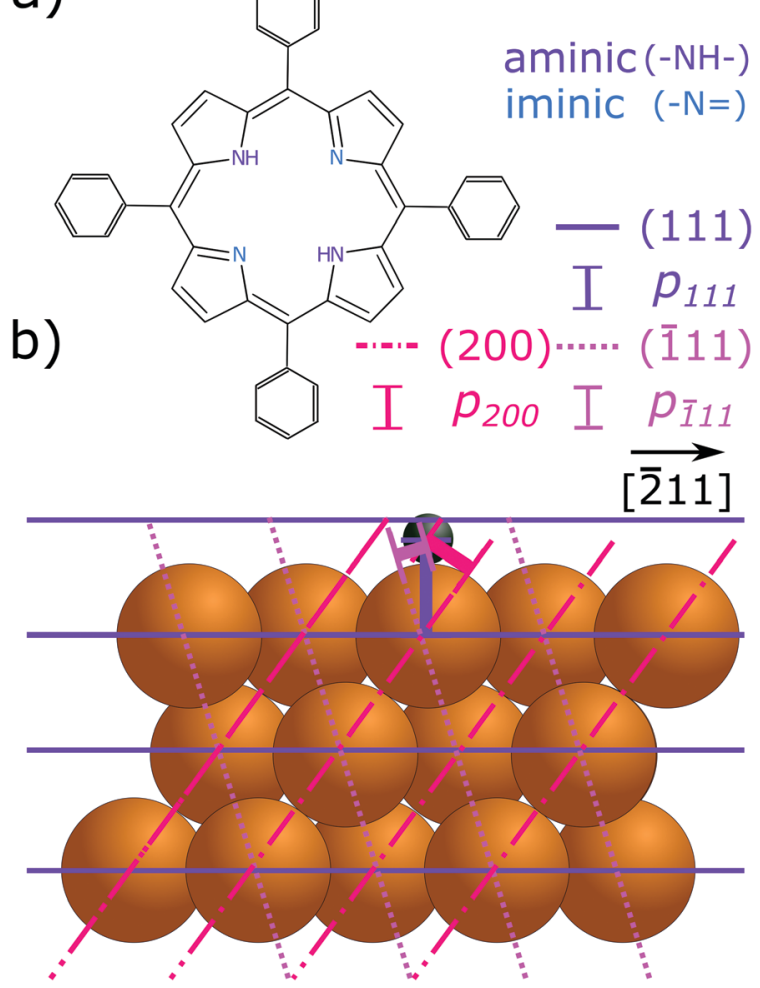

Fig. 1 (a) Schematic of $2 \mathrm{H}$-tetraphenyl porphyrin indicating the aminic and iminic $\mathrm{N}$ species. (b) Side view of the $\mathrm{Cu}(111)$ surface with the Bragg diffraction planes, corresponding to the (111), (111) and (200) Bragg reflections, overlaid, along with corresponding coherent position values $\left(p_{111}, p_{111}\right.$ and $\left.p_{200}\right)$ of an arbitrary adsorbed atom (black sphere) sitting atop the surface.

without significant distortions. Both studies, coupled with the radiation damage present in the study of Bürker et al., may thus throw uncertainty on the presence of this inverted model. This inverted model contravenes many expectations from gas phase and solvation chemistry, and is deeply counter-intuitive, thus we believe that it is important to provide detailed quantitative experimental structural data for a rigorous test of its correctness.
Specifically, we report new conclusive measurements of this system using the same NIXSW method, but carefully working under conditions that ensure beam damage was mitigated and also acquiring NIXSW data from two additional Bragg reflections, allowing determination of both the adsorption heights and the lateral adsorption sites of both $\mathrm{N}$ species. Our results thoroughly confirm the conclusions of Lepper et al. and the validity of the inverted adsorption structure for $2 H$-TPP.

The measurements were performed on the permanent end station of the I09 beam line at the Diamond Light Source (Oxfordshire, UK). ${ }^{17}$ Three surface reflections were used to generate the X-ray standing waves: the (111), (111) and (200) planes. To minimise radiation damage, the flux of the beam line was decreased by a factor of approximately 4 for the (111) reflection, and a factor of 6 for the (111) and (200) reflections, by offsetting the gap of the undulator away from the maximum of the emission curve for each required photon energy. $\mathrm{N} 1 \mathrm{~s}$ soft XPS (SXPS) data were recorded before and after the NIXSW measurements to monitor any remaining radiation damage (see Fig. S2 in the ESI $\dagger$ ), which was negligible. Further experimental details are given in the ESI. $\dagger$

The NIXSW technique ${ }^{14}$ exploits the X-ray standing wave that is created by the interference between the incident and reflected waves close to the Bragg condition for a given Bragg reflection $(h, k, l)$. The period of this standing wave matches the interplanar spacing $\left(d_{h k l}\right)$ between the Bragg diffraction planes. ${ }^{18}$ In the case of a homoatomic, face centred cubic crystal structure (e.g. pure $\mathrm{Cu}$ ) the Bragg diffraction planes are coincident with the atomic planes. The phase of the standing wave, and thus the location of the maximum intensity of the standing wave with respect to the Bragg diffraction planes, varies when the photon energy is scanned through the Bragg condition. When the phase is zero, the maximum intensity lies halfway between Bragg diffraction planes; when the phase is $\pi$ the maximum intensity is coincident with the Bragg diffraction planes. Any atom immersed in this standing wavefield will experience a varying electromagnetic field intensity as a function of its position between these diffraction planes, resulting in a characteristic absorption profile, which can be acquired by monitoring the photoelectron intensity profile. The measured profile is then
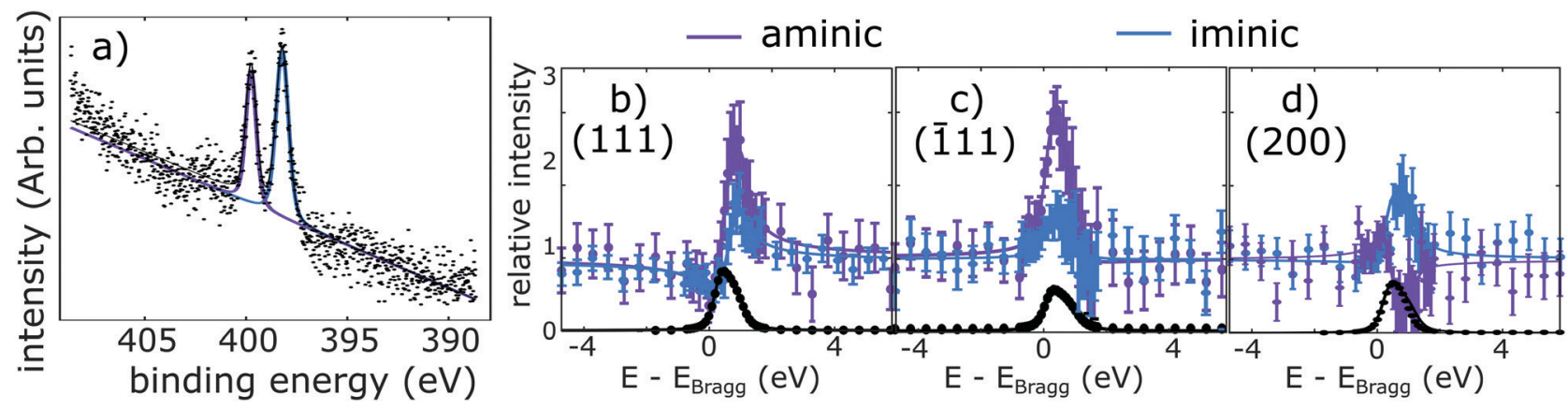

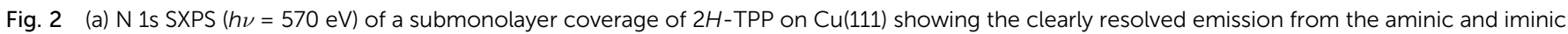

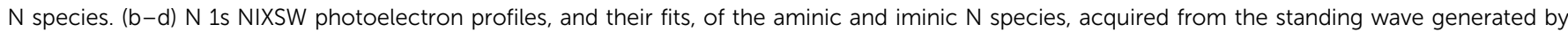

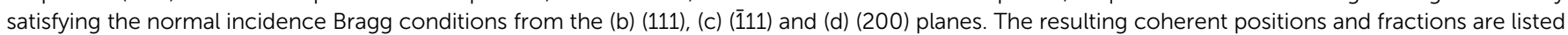
in Table 1. 
fitted uniquely, using dynamical diffraction theory, ${ }^{19}$ by two dimensionless parameters: ${ }^{14}$ the coherent fraction, $f_{h k l}$, and the coherent position, $p_{h k l}$. These are, respectively, the amplitude and phase of a Fourier transform component of the absorber location and broadly correspond to the degree of order and the mean position, respectively, of the absorber atoms relative to the Bragg diffraction planes. By recording NIXSW data at different Bragg reflections, $(h, k, l)$, we can use these amplitude and phase values to triangulate the position of the emitter atoms with respect to the underlying bulk crystal, as schematically demonstrated in Fig. 1 b.

The N 1s SXP spectrum for a ca. 0.5 monolayer coverage of $2 H$-TPP on $\mathrm{Cu}(111)$ is shown in Fig. 2a. This spectrum is consistent with the published data for this system. ${ }^{5,20,21}$ The higher binding energy species $(399.8 \mathrm{eV})$ is assigned to the aminic (-NH-) nitrogen atoms, the lower binding energy species $(398.2 \mathrm{eV})$ to the iminic $(-\mathrm{N}=)$ nitrogen atoms. The C 1s SXPS are shown in Fig. S3 in the ESI, $\dagger$ and consist of a primary peak with a notable shoulder at higher binding energy. In the previous work of Bürker et al., this shoulder at higher binding energy was assigned to $\mathrm{C}$ atoms bound to $\mathrm{N}$ atoms. Note that the primary $\mathrm{C}$ peak overlaps strongly the $\mathrm{C}$ peak due to a contaminant that was observed on the surface, also shown in Fig. S3 (ESI $\dagger$ ).

The $N$ 1s NIXSW data from the (111), (111) and (200) reflections, along with their associated fits, are shown in Fig. 2b-d (respectively). The corresponding coherent fractions and coherent positions are listed in Table 1. Our (111) data closely reproduce the results of Bürker et al., ${ }^{13}$ obtaining identical coherent positions and coherent fractions within the experimental uncertainty; this indicates that the beam damage that occurred during their experiment does not significantly alter the adsorption height of the $\mathrm{N}$ atoms. Our measured coherent positions correspond to adsorption heights, relative to the underlying $\mathrm{Cu}(111)$, of $1.88 \pm 0.08 \AA$ and $2.15 \pm 0.15 \AA$ for the iminic and aminic $\mathrm{N}$ species, respectively. At the (111) Bragg reflection both $\mathrm{N}$ species exhibit high coherent fractions, implying that all the $\mathrm{N}$ atoms of each species occupy closely similar heights above the surface and thus likely adsorb in only a single configuration (note a discussion on coverage and substrate temperature can be found in the ESI $\dagger$ ). In the (111) and (200) reflections, the aminic N species also exhibits a high coherent fraction, indicating that each of these atoms occupies a lateral position close to a site that shares the point group symmetry $(3 \mathrm{~mm})$ of the underlying substrate (i.e. atop a surface $\mathrm{Cu}$ atom or in a 3 -fold coordinated hollow site). The iminic $\mathrm{N}$ species, by contrast, exhibits lower coherent fractions in the

Table 1 Coherent fractions, $f_{h k l}$, and coherent positions, $p_{h k l}$, of the iminic and aminic $\mathrm{N}$ species for $2 \mathrm{H}$-TPP adsorbed on $\mathrm{Cu}(111)$. The values in brackets are the standard error at two standard deviations in the last significant figure of the quoted values

\begin{tabular}{lllllll}
\hline & $f_{111}$ & $p_{111}$ & $f_{\overline{1} 11}$ & $p_{\overline{1} 11}$ & $f_{200}$ & $p_{200}$ \\
\hline Iminic & $0.77(9)$ & $0.90(4)$ & $0.17(15)$ & $0.73(15)$ & $0.24(15)$ & $0.10(12)$ \\
Aminic & $0.78(16)$ & $0.03(7)$ & $0.77(13)$ & $0.34(6)$ & $0.82(17)$ & $0.73(8)$
\end{tabular}

(111) and (200) reflections, indicating that it must occupy sites of lower symmetry, qualitatively consistent with the prediction from Lepper et al. ${ }^{8}$ of adsorption in a bridge or near bridge site.

In our previous investigation of the adsorption of porphine on $\mathrm{Cu}(111)^{16}$ and cobalt porphine on $\mathrm{Cu}(111),{ }^{22}$ the centres of these molecules were found to occupy bridge sites with one of the molecular mirror planes coincident with the surface mirror plane that bisects the bridge site. In the determined $\mathrm{PhD}$ structure of porphine on $\mathrm{Cu}(111)^{16}$ the iminic $\mathrm{N}$ atoms occupy two adsorption sites that are mirrored across the [11ㄹ] direction, which is also a mirror plane of the substrate; by contrast, the mirror plane of the molecule that relates the two aminic $\mathrm{N}$ atoms lies in the [110] direction, which is not a mirror plane of the substrate. STM images of $2 H$-TPP on $\mathrm{Cu}(111)$ indicate this molecule adsorbs with the same azimuthal orientation as porphine; ${ }^{23}$ this same azimuthal orientation and adsorption site of the molecular centre is predicted in the DFT calculations of Lepper et al.

By assuming these two mirror conditions for the adsorption site of the iminic and aminic $\mathrm{N}$ atoms, we can calculate expected coherent positions and coherent fractions for different lateral nitrogen-substrate registry adsorption sites on the surface and compare them with the experimental measurements. In Fig. 3a and $\mathrm{b}$ are colour-scale maps on the $\mathrm{Cu}(111)$ surface that display the difference between these predicted and measured NIXSW parameters, expressed as a weighted mean-square error parameter as defined in the ESI, $\dagger$ as a function of possible lateral positions of the $\mathrm{N}$ atoms on the surface. These 2D maps are calculated at the height above the surface determined by the (111) coherent positions. Notice that the predicted values of the NIXSW parameters at different positions take account of different symmetrically-equivalent molecular orientations by summing over symmetrically equivalent substrate reflections (e.g., not only

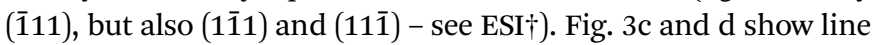
scans through these maps along the [110] and [112] direction for the iminic and aminic $\mathrm{N}$ species, respectively. Inspection of Fig. 3 clearly indicates that the iminic $\mathrm{N}$ atom occupies a bridge/ off-bridge site and the aminic $\mathrm{N}$ atoms occupy atop sites. The best fit to the experimental data corresponds to the iminic $\mathrm{N}$ atom sitting $1.1 \pm 0.2 \AA$ off atop.

Due to the coexistence of some degree of $\mathrm{C}$ contamination in our data, with an associated photoelectron binding energy similar to that of the primary $\mathrm{C}$ 1s peak, only NIXSW data from the shoulder in the C 1s spectra are considered here. The corresponding C 1s NIXSW data are shown in Fig. S4 in the ESI. $\dagger$ The resulting coherent fractions for this shoulder feature yielded a surprisingly high coherent fraction $(>0.4)$ across all three reflections (see Table S1 in $\mathrm{ESI} \dagger)$. Comparing the predicted adsorption site of $\mathrm{C}$ atoms bound to $\mathrm{N}$ (CN species) found in Lepper et al.'s calculations does result in a relatively high coherent fraction in the (111) reflection, but for the (111) and (200) reflections the expected coherent fractions are almost zero. This could be taken to cast some doubt on the inverted model, but the very good agreement with the $\mathrm{N}$ 1s data leads us to suspect that this $\mathrm{C}$ photoemission component corresponds to a set of $\mathrm{C}$ atoms other than those of the CN species. Such a shoulder is not reported for adsorption of $2 H$-TPP on $\mathrm{Ag}(111),{ }^{5}$ suggesting that 


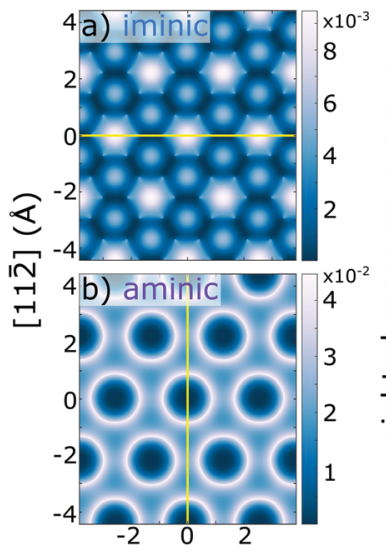

[11̄0] $(\AA)$

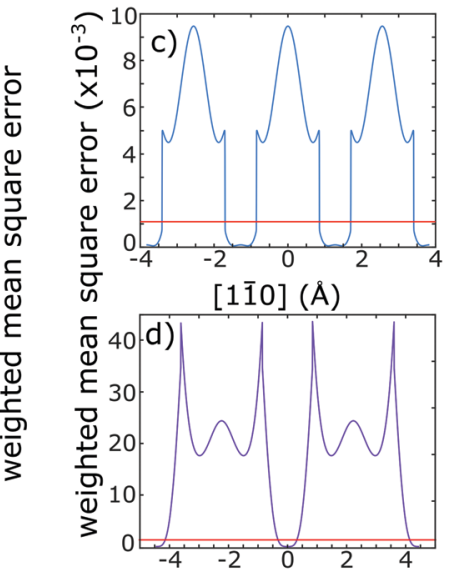

$[11 \overline{2}](\AA)$

Fig. 3 ( $a$ and b) 2D maps of the (111) surface in which the darkness of the colour-scale indicates the level of agreement between the measured NIXSW coherent positions and fractions for all Bragg scattering geometries and the values of these parameters expected for occupation of this lateral adsorption site for the (a) iminic and (b) aminic $\mathrm{N}$ species. The coordinate $(0,0)$ is atop a surface $\mathrm{Cu}$ atom, and the $2 \mathrm{D}$ planes are set at heights that correspond to measured $p_{111}$ values of 0.90 and 1.03 for the iminic and aminic $\mathrm{N}$ species, respectively. The calculation of the weighted mean square error is defined in eqn (S1) in the ESI. $\uparrow$ The closer the weighted mean square is to zero (dark blue/black), the better the site agrees with the experimental measurements. (c and d) 1D line scans of this parameter for the (c) iminic and (d) aminic N species taken along the [110] and [112]] surface directions that are indicated in panels (a) and (b) with a yellow line, respectively. Overlaid in (c) and (d) is the estimated uncertainty in the weighted mean square error (red line), which is the reciprocal of the sum of the squared reciprocals of the experimental uncertainty. Note that the best agreement with the experiment is for the iminic $\mathrm{N}$ atoms in an off-bridge site and for the aminic $\mathrm{N}$ atoms in a direct atop site.

the origin of this shoulder may arise from the contorted structure of the $2 H$-TPP on $\mathrm{Cu}(111)$. However, the $2 H$-TPP molecule contains over forty $\mathrm{C}$ atoms, so without more detailed understanding of the expected individual $\mathrm{C} 1 \mathrm{~s}$ chemical shifts for $2 H-\mathrm{TPP}$ on $\mathrm{Cu}(111)$, it is not possible to identify the site of these $\mathrm{C}$ atoms using the same methodology applied here for $\mathrm{N}$ atoms; the potential search parameter space is too complex. Note that we do not expect the $\mathrm{C}$ contamination to alter the adsorption structure of the $2 H$-TPP molecule. 2H-TPP molecules experience repulsive intermolecular interactions ascribed to the formation of a surface dipole, ${ }^{24}$ whereas adventitious carbon would be expected to form $\mathrm{C}$ clusters on the surface, ${ }^{25}$ limiting the likelihood of interaction.

The results of this investigation validate that $2 H$-tetraphenyl porphyrin adopts, upon adsorption on $\mathrm{Cu}(111)$, the so-called inverted conformation, shown in Fig. S1 in the ESI, $\dagger$ with the iminic nitrogen atoms lying in bridging, rather than off-atop sites. The level of agreement between the experimental results presented here, and the DFT calculations of Lepper et al., ${ }^{8}$ is excellent. The iminic nitrogen atoms were determined to adsorb in sites displaced laterally by $1.1 \pm 0.2 \AA$ from $\mathrm{Cu}$ atop sites, whereas the aminic $\left(-\mathrm{NH}^{-}\right)$nitrogen atoms were determined to adsorb directly atop $(0.0 \pm 0.4 \AA)$ nearest surface $\mathrm{Cu}$ atom. For an intact molecule, this agrees best with the adsorbed molecule being centred above a surface bridge site.

We acknowledge Diamond Light Source for the award of beam time (SI24113-1), the Deutsche Forschungsgemeinschaft (DFG) for support through the Germany's Excellence Strategy (e-conversion Cluster of Excellence EXC 2089/1-390776260) and the TUM International Graduate School of Science and Engineering (IGSSE), GSC 81. PTPR would like to thank the Advance Characterisation of Materials (ACM) CDT.

\section{Conflicts of interest}

There are no conflicts to declare.

\section{Notes and references}

1 J. M. Gottfried, Surf. Sci. Rep., 2015, 70, 259-379.

2 W. Auwärter, D. Écija, F. Klappenberger and J. V. Barth, Nat. Chem., 2015, 7, 105.

3 K. Diller, A. C. Papageorgiou, F. Klappenberger, F. Allegretti, J. V. Barth and W. Auwärter, Chem. Soc. Rev., 2016, 45, 1629-1656.

4 Ö. Birel, S. Nadeem and H. Duman, J. Fluoresc., 2017, 27, 1075-1085.

5 K. Diller, F. Klappenberger, M. Marschall, K. Hermann, A. Nefedov, C. Wöll and J. Barth, J. Chem. Phys., 2012, 136, 014705.

6 W. Auwärter, K. Seufert, F. Klappenberger, J. Reichert, A. WeberBargioni, A. Verdini, D. Cvetko, M. Dell'Angela, L. Floreano, A. Cossaro, G. Bavdek, A. Morgante, A. P. Seitsonen and J. V. Barth, Phys. Rev. B: Condens. Matter Mater. Phys., 2010, 81, 245403.

7 S. P. Jarvis, S. Taylor, J. D. Baran, D. Thompson, A. Saywell, B. Mangham, N. R. Champness, J. A. Larsson and P. Moriarty, J. Phys. Chem. C, 2015, 119, 27982-27994.

8 M. Lepper, J. Köbl, T. Schmitt, M. Gurrath, A. de Siervo, M. A. Schneider, H.-P. Steinrück, B. Meyer, H. Marbach and W. Hieringer, Chem. Commun., 2017, 53, 8207-8210.

9 F. Albrecht, F. Bischoff, W. Auwärter, J. V. Barth and J. Repp, Nano Lett., 2016, 16, 7703-7709.

10 J. C. Moreno-López, D. J. Mowbray, A. Pérez Paz, R. C. de Campos Ferreira, A. Ceccatto dos Santos, P. Ayala and A. de Siervo, Chem. Mater., 2019, 31, 3009-3017.

11 D. P. Woodruff, Jpn. J. Appl. Phys., 2019, 58, 100501.

12 A. Michaelides, K. Reuter and M. Scheffler, J. Vac. Sci. Technol., A, 2005, 23, 1487-1497.

13 C. Bürker, A. Franco-Cañellas, K. Broch, T.-L. Lee, A. Gerlach and F. Schreiber, J. Phys. Chem. C, 2014, 118, 13659-13666.

14 D. Woodruff, Rep. Prog. Phys., 2005, 68, 743.

15 K. Diller, R. J. Maurer, M. Müller and K. Reuter, J. Chem. Phys., 2017, 146, 214701.

16 D. A. Duncan, P. Casado Aguilar, M. Paszkiewicz, K. Diller, F. Bondino, E. Magnano, F. Klappenberger, I. Píš, A. Rubio, J. V. Barth, A. Pérez Paz and F. Allegretti, J. Chem. Phys., 2019, 150, 094702.

17 T.-L. Lee and D. A. Duncan, Synchrotron Radiation News, 2018, 31, 16-22.

18 M. J. Bedzyk and G. Materlik, Phys. Rev. B: Condens. Matter Mater. Phys., 1985, 32, 6456-6463.

19 B. W. Batterman, Phys. Rev., 1964, 133, A759.

20 H. Marbach, Acc. Chem. Res., 2015, 48, 2649-2658.

21 J. Xiao, S. Ditze, M. Chen, F. Buchner, M. Stark, M. Drost, H.-P. Steinrück, J. M. Gottfried and H. Marbach, J. Phys. Chem. C, 2012, 116, 12275-12282.

22 M. Schwarz, M. Garnica, D. A. Duncan, A. Pérez Paz, J. Ducke, P. S. Deimel, P. K. Thakur, T.-L. Lee, A. Rubio, J. V. Barth, F. Allegretti and W. Auwärter, J. Phys. Chem. C, 2018, 122, 5452-5461.

23 F. Buchner, J. Xiao, E. Zillner, M. Chen, M. Röckert, S. Ditze, M. Stark, H.-P. Steinrück, J. M. Gottfried and H. Marbach, J. Phys. Chem. C, 2011, 115, 24172-24177.

24 G. Rojas, X. Chen, C. Bravo, J.-H. Kim, J.-S. Kim, J. Xiao, P. A. Dowben, Y. Gao, X. C. Zeng, W. Choe and A. Enders, J. Phys. Chem. C, 2010, 114, 9408-9415.

25 T. Niu, M. Zhou, J. Zhang, Y. Feng and W. Chen, J. Am. Chem. Soc., 2013, 135, 8409-8414. 